\title{
Gut Microbiome and their metabolic impact: New frontier
}

\author{
Dipendra Raj Pandeya ${ }^{1,2}$
}

\author{
Affiliations: \\ ${ }^{1}$ Department of Clinical Laboratory Sciences, College of \\ Applied Medical Sciences, Al Jouf University, Al Qurayyat, \\ Saudi Arabia
}

${ }^{2}$ Department of Clinical Biochemistry, College of Medicine, Nepalese Army Institute of Health Sciences, Kathmandu, Nepal

\section{Correspondence to:}

Dr. Dipendra Raj Pandeya, PhD

Department of Clinical Laboratory Science, College of Applied Medical Sciences,

Al Jouf University

Al Qurayyat, Saudi Arabia

Email: dipendra100@yahoo.com

How to cite this article:

Gut Microbiome and their metabolic impact: New frontier. Ann. Clin. Chem. Lab. Med. 2016:2(2); 1-3

DOI: http://dx.doi.org/10.3126/acclm.v2i2.17068

(C) 2016 Nepalese Association for Clinical Chemistry

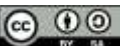

This work is licensed under a Creative Commons Attribution-Share Alike 4.0 International License.
The human gastrointestinal tract is inhabitation of over 100 trillion microorganisms, the gut microbiota and shares our bodies not as invaders, but as integral parts of ourselves [1]. Our adult bodies shield $\sim 10$ times more microbial than human cells and this reflects that we humans are mostly microbes. The human microbiome refers to the assemblage of microbes that live in the human body and are inhabit all parts of our body that are exposed to the environment, but most reside within the gastrointestinal tract, where the bacterial density reaches $10^{11}-10^{12}$ cells/g in the distal human colon [2]. Starting from sterile foetal life to exposure to microbes first occurs during birth from mother and surrounding environment colonizes the infant's gut [3]. Humans have coevolved with their microbes as a physiologic community over thousands of years, but this relationship, is now being dramatically affected by shifts in the collective human microbiome resulting from changes in the environment and societal norms. The distribution and density of gut microbiota habitants fluctuate markedly within an individual and between individuals and is modulated by diverse determinants of temporal and spatial variability. There are numerous external factors that have potential to influence the microbial composition in the gut are host genetics [4], birth delivery mode [5], geographical impacts [6], influence of ageing [7], influence of diet [8], impact of antibiotics [9], and pre- and probiotics [10]. These microbes have tremendous potential to impact our physiology with Protective functions such as i) combating opportunistic pathogens, ii) nutrient and receptor competition with pathogen, iii) production of antimicrobial factors e.g. bacteriocins, lactic acids and Structural function including 1) barrier fortification, 2) development of gutassociated lymphoid tissues (GALTs), 3) development of the immune system. Equally important are the metabolic function such as 1) Control intestinal epithelial cell differentiation and proliferation, 2) detoxification of potentially harmful xenobiotics, 3) synthesis of essential amino acids and vitamins e.g. vitamin $\mathrm{K}$, thiamine, riboflavin, biotin and folate, 4) metabolization of energy sources that are otherwise inaccessible to their hosts such as cellulose and resistant starches eg iron absorption, salvage of energy etc.

Because of the magnitude of the impact microorganisms have on humans, many researchers have begun referring to a human "superorganism"[11]. So, significant technological advances have facilitated the characterization of key gut microbial populations, providing information that can be harnessed in a variety of ways from the laboratory bench to the patient's bedside [12]. Many scientists noted the considerable genetic signal from microbes in the body and the existence of technology to analyze these 
microorganisms. Large and diverse populations of bacteria, viruses, and fungi occupy almost every surface of the human body [13]. It is estimated that there are nearly 30 trillion bacterial cells living in or on each human [14]. It was recently demonstrated that the microbiome, which represents the collective genomes of the gut microbiota, is approximately 150 times larger than the human gene complement, with an estimated set of 3.3 million microbial genes [15] and it may weigh as much as five pounds. The human genome is predicted to contain 22,000 coding genes, but more than 200,000 proteins are expressed in human cells. The individual genetic signatures of the microbiomes vary widely between individuals. The combine of the microbiome and the human genome is termed the metabolome [16]. All genes acquaint in the body have the likely to affect human health, either positively or negatively [17]. Therefore, because the metabolome consists of mainly genes from these microbes, it can be said that human beings only genetically inherit $1 \%$ of the genes that reside in cells in their body.

Ascertaining the functional imputes of the microbiome is crucial for interpreting their role on host metabolism and disease [18]. So, the advent of new molecular techniques like high- throughput sequencing techniques such as 16s rRNA sequencing and Shotgun metagenome sequencing has helped to reveal the complexity and composition of the gut microbiota. [19]. Metatranscriptomics, metaproteomics, and metabonomics will be useful to explore the functional aspects of the gut microbiome from the top down. Real time analysis of the intestinal microbiome is a useful tool in the development of personalized approaches to targeted therapies. The combination of metabolic profiling and metagenomic studies of gut microbiota permits the study of host and microbial metabolism in great detail. Phylogenetic analysis has suggested that most members of the gut belong to the phyla Firmicutes and Bacteroidetes, with lower abundances of Proteobacteria, Verrucomicrobia, Actinobacteria, Fusobacteria, and Cyanobacteria [20,21]. The Firmicutes and the Bacteroidetes dominated, together accounting for $>98 \%$ of all $16 \mathrm{~S}$ rRNA sequences in each mammalian host. Therefore, dominant groups would monopolize the gene switching market and entrants would be rare and have discrete and complementary metabolic roles within the community.

Although scientists are increasingly switching their attention toward studying not just what microbes are present in (and on) the human body, but also what those microbes are doing and their interaction with the body cells, the field still revolves around genomics. A major goal of the Human Microbiome Project (HMP) is to characterize the genomic makeup of all microbes inhabiting the human body. Without understanding the interactions between our human and microbial genomes, it is impossible to obtain a complete picture of our biology.
Both good and bad bugs are present in our body and there should be homeostasis maintained between these two groups which are vital to the maintenance of human health but when mutualistic or commensal bacteria are replaced or outcompeted by less favorable or pathogenic species, dysbiosis can occur. So, dysbiosis leads to disease such as irritable bowel syndrome (IBS), inflammatory bowel disease (IBD), respiratory and chronic pulmonary disease, immunological impairment, colorectal cancer, metabolic diseases, bacterial infections and cardiovascular risks, etc. along all life stages.

In the past decade, the field of microbiota research has exploded, resulting in the publication of a plethora of reports that describe both the individual members of our intestinal microbiota and their wide-ranging impact on host physiology. Disease-causing microbes accumulate over time, changing gene activity and metabolic processes and resulting in an abnormal immune response against substances and tissues normally present in the body. Given the serious burden obesity and its comorbidities cardiovascular disease and diabetes puts on society, there is a pressing need to find new ways of tackling this problem. Investigating the role of the gut microbiota in metabolic diseases is one important way to address this challenge. So, Conventionalization studies, studies in germ-free mice, and studies using antibiotics yield new strategies to improve health by modifying the gut microbiota (e.g., pre- and probiotics and fecal transplantation) have attracted increasing attention [22]. However, the kinship between host and microbe is now being endangered by drastic changes in the environment, diet, and life style over the past few years which have almost definitely remolded the collective human gut microbiome. It is thought that every person's microbiome is slightly different. In fact, work is underway to investigate the use of microbiome to identify individuals, much like fingerprints [23]. Nearly every scientific study performed that has attempted to correlate the microbiome with specific traits or diseases has been successful. In other words studies are finding that our bacteria can be linked to or associated with: obesity, malnutrition, heart disease, diabetes, celiac disease, eczema, asthma, multiple sclerosis, colitis, some cancers, and even autism. Using new technologies and borrowing strategies from other disciplines, we are learning what constitutes a healthy microbiome and starting to see how changes in our personal ecosystems can affect our health.

If a clear causal relationship between the gut microbiota and metabolic health can be established:

- The microbiome is inherent to human physiology, health, and disease.

- The microbiome may find out how a person reacts to a drug treatment.

- Given the emerging nature of research on the microbiome, genetic studies that assess the relative abundance of different species in the human microbiome have linked various 
- combinations of microbe species to certain human health conditions.

- Dietary interferences specified to have an impact on host biology via their impact on the microbiome are being developed, and the market for these products is seeing tremendous success.

- A more accomplished understanding of the assortment of microbes in the human microbiome could instigate to new treatments

Gut microbiota changes and therapeutic modulation of microbiota are having potential value in the future for management of different diseases. It has been shown that the dysbiosis of these microbes may lead to obesity and other health complications. The understanding of how exactly the gut microbiota affects human health will require further studies. In addition, the intervention studies, possibly using findings from case-control and prospective studies, to alter a disease related microbiota to a healthy state. A combination of microbial sequencing and animal experiments may provide further insights into how the gut microbiota affects host metabolism and physiology and will aid in the diagnosis of microbiome related diseases and could potentially provide new means to prevent disease onset or to improve prognosis. The field of gut microbiome is rapidly developing and we expect that it will continue in the same pace in the years to come and the outcomes will help us to keep our life healthy.

\section{References}

1. Costello EK, Lauber CL, Hamady M, Fierer N, Gordon JL, Knight R. Bacterial community variation in human body habitats across space and time. Science 2009; 362:16947.

2. Backed F, Ley RE, Sonnenbuurg, JL, Peterson, DA, Gordon JI. Science 2005; 307:1915-20.

3. Li J, Jia H, Cai X, Zhong H, Feng $Q$, Sunagawa $S$, et al. An integrated catalog of reference genes in the human gut microbiome. Nat Biotechnol. 2014;32:834-41.

4. Zoetendal, E.G., Akkermans, A.D.L., Akkermans-van Vliet, W.M., de Visser, J.A.G.M. \& De Vos, W.M. (2001a). The Host Genotype Affects the Bacterial Community in the Human Gastrointestinal Tract. Microbial Ecology in Health and Disease 13, 129-134.

5. Grönlund, M.M., Lehtonen, O.P., Eerola, E. \& Kero, P. (1999). Fecal microflora in healthy infants born by different methods of delivery: permanent changes in intestinal flora after cesarean delivery. Journal of Pediatric Gastroenterology and Nutrition 28(1), 19-25.

6. Genta, R.M., Gurer, I.E. \& Graham, D.Y. (1995). Geographical pathology of Helicobacter pylori infection: is there more than one gastritis? Annals of Medicine 27(5), 595-599.

7. Woodmansey, E.J. (2007). Intestinal bacteria and ageing. Journal of Applied Microbiology 102(5), 1178-1186.
8. Balmer, S.E. \& Wharton, B.A. (1991). Diet and faecal flora in the newborn: iron. Archives of Disease in Childhood 66(12), 1390-1394.

9. Jernberg, C., Löfmark, S., Edlund, C. \& Jansson, J.K. (2007). Long-term ecological impacts of antibiotic administration on the human intestinal microbiota. ISME Journal 1(1), 56-66.

10. Fuller, R. (1986). Probiotics. Society for Applied Bacteriology Symposium Series 15, 1S-7S.

11. Sleator RD. Probiotic therapy - recruiting old friends to fight new fo es. Gut Pathog. 2010 Jun 25;2(1):5

12. Mani S, Boelsterli UA, Redinbo, MR. Understand and modulating mammalian microbial communication for improved human health. Annu Rev Pharmacol Toxicol 2014;54:559-80.

13. Ursell LK, Metcalf JL, Parfrey LW, Knight R. Defining the Human Microbiome. Nutrition reviews. 2012;70(1): S38-S44.

14. Sender R, Fuchs S, Milo R. Revised Estimates for the Number of Human and Bacteria Cells in the Body. PLoS Biol. 2016 Aug 19;14(8):e1002533.

15. Qin J, Li R, Raes J, et al. A human gut microbial gene catalogue established by metagenomic sequencing. Nature. 2010;464:59-65.

16. Wall, R., Ross, R. P., Shanahan, F., O'Mahony, L., O'Mahony, C., Coakley, M., Stanton, C. Metabolic activity of the enteric microbiota influences the fatty acid composition of murine and porcine liver and adipose tissues. The American Journal of Clinical Nutrition. 2009; 89(5),1393-1401.

17. McNulty, N. P, Wu, M, Erickson, AR, Pan, C, Erickson, BK, Martens, EC, Gordon, Jl. Effects of Diet on Resource Utilization by a Model Human Gut Microbiota Containing Bacteroides cellulosilyticus WH2, a Symbiont with an Extensive Glycobiome. Plos Biology. 2013;11(8), e1001637.

18. Joice R, Yasuda K, Shafquat A, Morgan XC, Huttenhower C. Determining microbial products and identifying melocular targets in the human microbiome. Cell Meatb. 2014;20:731-41.

19. Moschen AR, Wieser V, Tilg H. Dietary factors: Major regulators of the gut's microbiota. Gut Liver. 2012;6:411-16.

20. Hugenholtz, P., Goebel, B.M., and Pace, N.R. 1998. Impact of culture-independent studies on the emerging phylogenetic view of bacterial diversity. J. Bacteriol. 180: 4765-4774.

21. Human Microbiome Project Consortium. A framework for human microbiome research. Nature. 2012; 486: 215-21.

22. Schnabl B, Brenner DA. Interactions between the intestinal microbiome and liver diseases. Gastroenterology 2014;146:1513-24.

23. Franzosa EA, Huang $K$, Meadow JF, Gevers D, Lemon $\mathrm{KP}$, Bohannan BJ, Huttenhower C. Identifying personal microbiomes using metagenomic codes. Proc Natl Acad Sci U S A. 2015;112(22):E2930-8 